\title{
Via Póstero-Interna na Fixação de Fragmento Posterior em Fratura Complexa do Pilão Tibial
}

\section{Posteromedial Approach for Fixation of Posterior Fragment in Complex Tibial Pilon Fracture}

Carla Martins ${ }^{1}$, Miguel Varzielas ${ }^{1}$, João Morais ${ }^{1}$, Eduardo Mendes ${ }^{1}$

\section{RESUMO}

As fraturas do maléolo posterior são frequentes nas fraturas do tornozelo. A sua fixação é importante para a estabilidade sindesmótica e desempenha um papel importante na prevenção da artrose pós-traumática e, consequentemente, leva a um melhor resultado funcional. A qualidade de redução é melhor se utilizada uma via posterior em relação às técnicas habituais.

Reportamos o caso de um homem de 52 anos com uma fratura do pilão tibial AO/OTA 43C3 e Bartonicek tipo 3. A redução anatómica e fixação do maléolo posterior foi realizada através de uma abordagem póstero-interna, com bons resultados funcionais 9 meses de pós-operatório.

A abordagem póstero-interna constitui uma via segura para redução e fixação do maléolo posterior que permite também a redução e fixação da restante tíbia distal, permitindo uma rápida recuperação funcional e minimizando complicações degenerativas tardias.

PALAVRAS-CHAVE: Fixação Interna de Fraturas/métodos; Fraturas da Tíbia/cirurgia; Fraturas da Tíbia/diagnóstico por imagem 


\section{ABSTRACT}

Fractures of the posterior malleolus are common in ankle fractures. Its fixation is important for syndesmotic stability and plays an important role in the prevention of post-traumatic arthrosis and, consequently, leads to a better functional outcome. Reduction quality is best if you use a backtrack compared to usual techniques.

We report the case of a 52-year-old male with a tibial pilon fracture AO/OTA 43C3 and Bartonicek type 3. The anatomical reduction and fixation of the posterior malleolus was performed through a posteromedial approach, with good functional results 9 months postoperatively.

The posteromedial approach is a safe way to reduce and fix the posterior malleolus, which also allows the reduction and fixation of the remaining distal tibia, allowing a rapid functional recovery and minimizing late degenerative complications.

KEYWORDS: Fracture Fixation, Internal/methods; Tibial Fractures/diagnostic imaging; Tibial Fractures/surgery

\section{INTRODUÇÃO}

As fraturas do tornozelo compreendem aproximadamente $9 \%$ de todas as fraturas. As fraturas do pilão são lesões graves que afetam a superfície articular da tíbia distal. ${ }^{1}$ Algumas lesões resultam de um trauma de alta energia, com carga axial, enquanto outras tendem a ser o resultado de um trauma de baixa energia devido a um mecanismo de rotação. ${ }^{2}$ Por sua vez, uma fratura do maléolo posterior ocorre em cerca de 46\% das fraturas do tornozelo Weber tipo B e C. A diferença entre fraturas do maléolo posterior e fraturas posteriores do pilão tibial é apenas uma convenção e defende-se que fragmentos do maléolo posterior que compreendam mais de 50\% da articulação sejam considerados fraturas do pilão tibial. ${ }^{3}$ De acordo com alguns autores, fragmentos de mais de $25 \%$ da superfície articular posterior da tíbia distal no plano sagital devem ser tratados cirurgicamente. ${ }^{4}$ Novas ideias sobre anatomia, tratamento e resultados têm questionado esta recomendação. ${ }^{3,5}$

Para alcançar uma boa função, devemos restaurar a superfície articular através de uma redução anatómica ideal e de uma fixação estável. Recentemente, o tratamento das fraturas do maléolo posterior e do pilão ganhou crescente atenção. Pequenas fraturas do rebordo tibial posterior foram frequentemente ignoradas ou fixadas usando um parafuso compressivo ântero-posterior sem redução apropriada obtendo resultados inferiores. ${ }^{6-8}$ A fixação do maléolo posterior é importante para a estabilidade sindesmótica: restaura a integridade da incisura fibular da tíbia e facilita a redução anatómica do perónio. Além disso, há evidências crescentes do importante papel do maléolo posterior no desenvolvimento da artrose pós-traumática. ${ }^{9-11} \mathrm{Com}$ um maléolo posterior deslocado, o centro do stress muda de forma anterior e interna na superfície articular. ${ }^{12}$ Posteriormente, as fraturas maleolares posteriores viciosamen- te consolidadas levam a um desfecho funcional mau e a uma má qualidade de vida. ${ }^{\circledR}$ Ainda não há consenso no que se refere ao tamanho exato dos fragmentos de maléolo posterior que justificam redução aberta e fixação interna (RAFI). Fragmentos maiores do que 5\% da superfície articular tibiotalar com um desnível articular de pelo menos $1 \mathrm{~mm}$, progridem mais frequentemente em direção à artrose, que aqueles sem desnível. 5,13

Bartoníček et al introduziu uma classificação para as fraturas do maléolo posterior com base na tomografia computadorizada (TC) nos planos transversal, sagital e frontal e reconstruções 3D. Esta classificação refere-se principalmente ao envolvimento da incisura. Recomendam RAFI para todos os grandes fragmentos triangulares póstero-externos, qualquer fragmento intercalar desviado, fragmentos póstero-externos menores com desvio da incisura e fragmentos póstero-internos de duas partes. Para conseguir isso, geralmente é necessária a RAFI através de uma abordagem póstero-externa ou póstero-interna. Uma vez que a abordagem póstero-interna é usada com menos frequência, mas pode ser muito útil especialmente para fragmentos póstero-internos, nesta nota técnica discutiremos as indicações, técnica, benefícios e limitações.

\section{CASO CLÍNICO}

Um homem de 52 anos, sem antecedentes relevantes, sofreu uma queda em contexto de acidente de trabalho da qual resultou traumatismo do tornozelo esquerdo. Ao exame objetivo apresentava edema e dor à palpação da região da perna e tornozelo. Ausência de feridas e sem alterações neurovasculares. Realizou radiografia e TC (Fig. 1) que revelaram fratura do pilão tibial, $43 \mathrm{C} 3$ segundo a classificação AO/OTA e tipo 3 (fragmento póstero-interno em duas partes), segundo a classificação de Bartonicek et al para a fratura do maléolo posterior. 

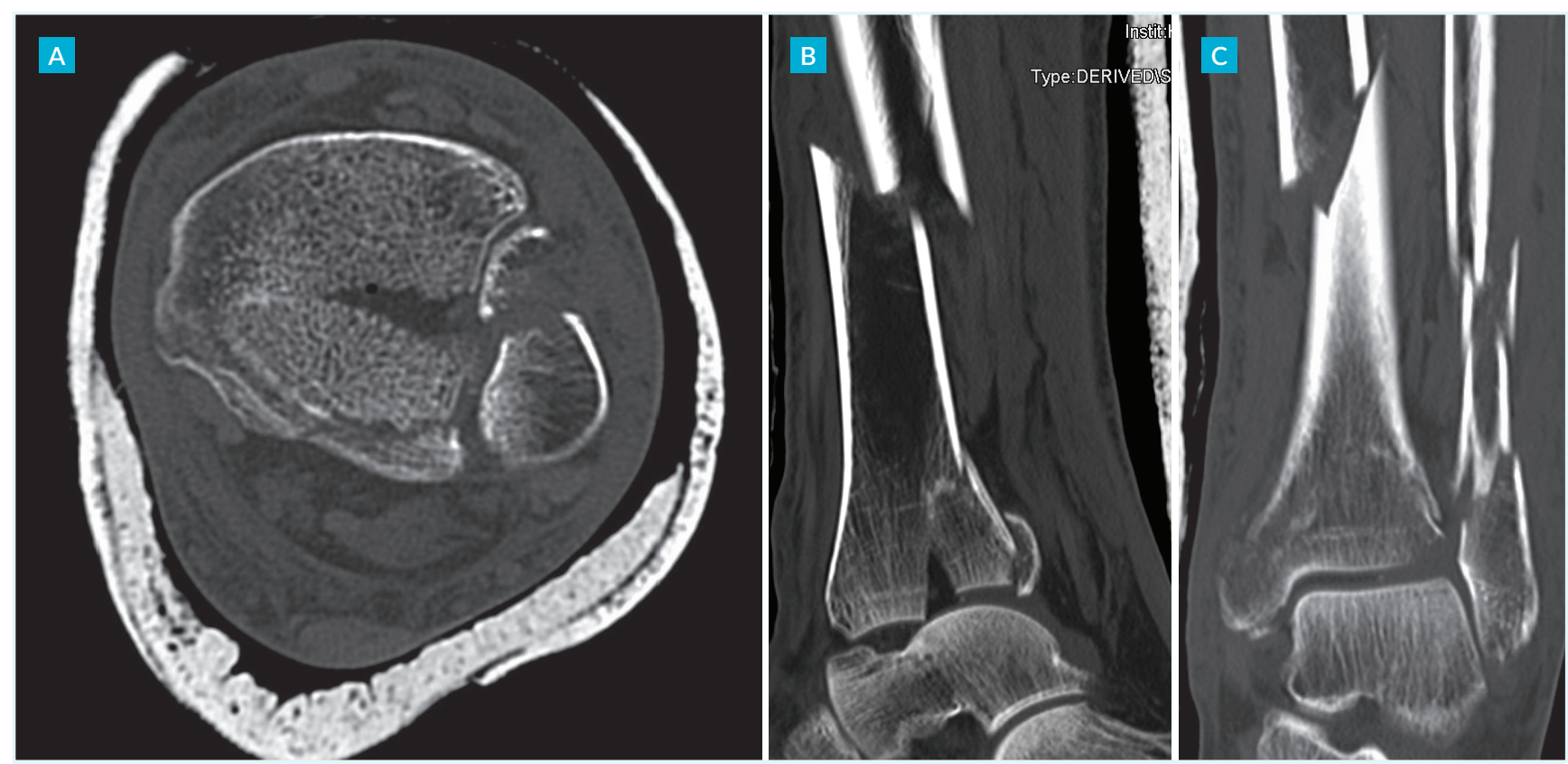

FIGURA 1. Imagens de TC pré-operatório do paciente com fratura do pilão tibial: Fragmento intercalar e incongruência da incisura fibular da tíbia. Vista axial (A), sagital (B), coronal (C).

Foi submetido a redução aberta e fixação interna (Fig. 2). No maléolo peronial usou-se abordagem externa direta com redução e colocação de 2 parafusos compressivos interfragmentários e placa LCP. Para abordagem da tíbia distal, usou-se uma abordagem póstero-interna. Fez-se redução e fixação do maléolo posterior com 2 parafusos compressivos interfragmentários e colocação de placa LCP em batente. Pela mesma abordagem foi feita redução do pilão tibial e colocação de placa LCP interna.

A abordagem póstero-interna da tíbia distal geralmente é efetuada com o doente em decúbito ventral, com uma almofada sob o pé, de maneira a otimizar o acesso. 0 uso de garrote é recomendado para prevenção de sangramento excessivo e visualização adequada durante a cirurgia. A incisão é feita $3 \mathrm{~cm}$ acima da tuberosidade do calcâneo, entre o tendão de Aquiles e o bordo posterior do maléolo medial. O prolongamento da incisão é ditado pela extensão do envolvimento metafisário. A dissecção é feita através dos compartimentos posteriores (superficial e profundo), evitando dissecção para além do tecido peritendinoso do tendão de Aquiles. A dissecção progride pelo intervalo entre o rolo vasculonervoso (artéria e nervo tibial posterior) e o longo flexor do hallux. Faz-se incisão do periósteo, expõe-se parcialmente a articulação tibiotalar e a incisura fibular da tíbia, procedendo-se em seguida à redução e fixação da fratura. Após a fixação, a estabilidade da sindesmose é testada com rotação externa, colocando-se um parafuso sindesmótico, caso necessário.

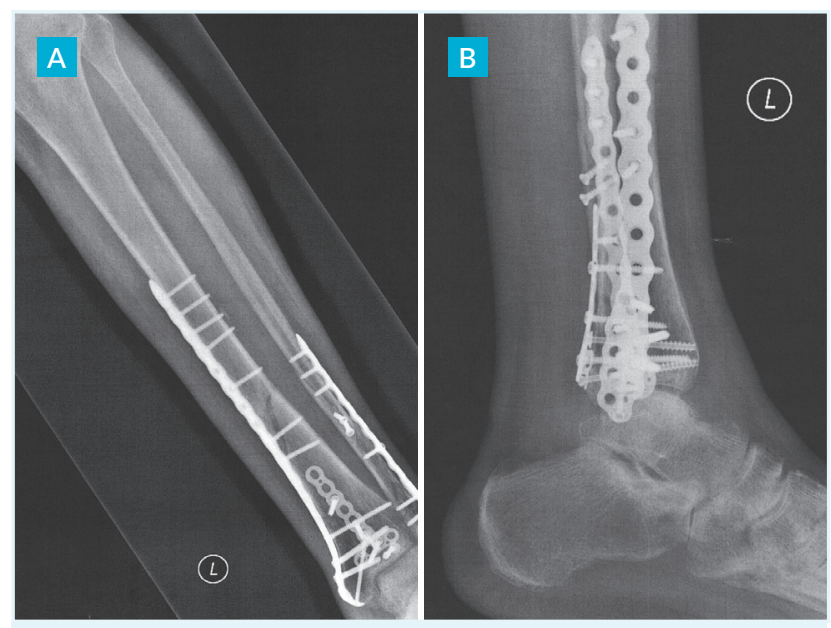

FIGURA 2. Imagens do pós-operatório, radiografia anteroposterior (A) e lateral (B).

\section{DISCUSSÃO}

As fraturas do tornozelo são fraturas frequentes. Contudo, existe ainda alguma controvérsia na literatura quanto ao seu tratamento. Ao longo das últimas décadas, o seu tratamento evoluiu com o desenvolvimento de diferentes estratégias e abordagens cirúrgicas. ${ }^{5,3,9-11,14}$ O tratamento tem por objetivo restaurar a congruência articular e o alinhamento mecânico de modo a incentivar a reabilitação funcional precoce. A abordagem é principalmente determinada pelo padrão individual da lesão articular, pelas opções de fixação interna e pelas lesões que acompanham a fratura do tornozelo. Quanto mais complexo o padrão de fratura, maior a necessidade de uma abordagem que visualize mais do que uma co- 
luna (lateral, posterior e medial). Na literatura são descritas duas abordagens posteriores principais: a abordagem póstero-interna e póstero-externa.A abordagem póstero-interna permite uma boa visão dos fragmentos posteriores com extensão mediana. Obtém-se um acesso direto à superfície articular do maléolo posterior e da incisura angular da tíbia, o que facilita a redução direta de fragmentos intercalares, conferindo-Ihe desta forma vantagens em comparação com a abordagem póstero-externa. ${ }^{9,14}$ A qualidade da redução anatómica da superfície articular tibial é o fator mais importante no tratamento dessas fraturas, correlacionando-se com melhores resultados funcionais.

Em resumo, a abordagem póstero-interna é elegante e direta no tratamento de fraturas póstero-mediais do pilão, fraturas maleolares posteriores com extensão medial ou fragmentos intercalares. Além disso, as complicações pós-operatórias são limitadas desde que o rolo neurovascular póstero-medial seja respeitado. No presente caso, esta abordagem, permitiu uma melhor redução da fratura, com resultados favoráveis.

CONFLITOS DE INTERESSE: Os autores declaram não ter qualquer conflito de interesse na realização do presente trabalho.

FONTES DE FINANCIAMENTO: Não houve qualquer fonte de financiamento na realização do presente trabalho.

CONFIDENCIALIDADE DOS DADOS: Os autores declaram ter seguido os protocolos da sua instituição acerca da publicação dos dados de doentes.

PROTEÇÃO DE PESSOAS E ANIMAIS: Os autores declaram que os procedimentos seguidos na elaboração do presente trabalho estão em conformidade com as normas das comissões de investigação clínica e de ética, bem como da declaração de Helsínquia e da Associação Médica Mundial.

CONFLICTS OF INTEREST: The authors declare that they have no conflicts of interest.

FINANCIAL SUPPORT: This work has not received any contribution, grant or scholarship.

CONFIDENTIALITY OF DATA: The authors declare that they have followed the protocols of their work center on the publication of data from patients.

PROTECTION OF HUMAN AND ANIMAL SUBJECTS: The authors declare that the procedures followed were in accordance with the regulations of the relevant clinical research ethics committee and with those of the Code of Ethics of the World Medical Association (Declaration of Helsinki).

\section{REFERÊNCIAS}

1. Court-Brown CM, Caesar B. Epidemiology of adul fractures: a review. Injury. 2006;37:691-7. doi:10.1016/j.injury.2006.04.130.

2. Korkmaz A, Ciftdemir M, Ozcan M, Copuro glu C, Sarıdo gan $K$. The analysis of the variables, affecting outcome in surgically treated tibia pilon fractured patients. Injury. 2013;44:1270-4. doi: 10.1016/j.injury.2013.06.016.

3. Bartoníček J, Rammelt S, Kostlivý K, Vaněček V, Klika D, Trešl I. Anatomy and classification of the posterior tibial fragment in ankle fractures. Arch Orthop Trauma Surg. 2015;135:506-16. doi: 10.1007/s00402-015-2171-4.

4. Muller ME, Nazarian S, Koch P, Schatzker J. The Comprehensive Classification of Fractures of Long Bones. Berlin: Springer; 1987.

5. Heim D, Niederhauser K, Simbray N. The Volkmann dogma: a retrospective, long-term, single-center study. Eur J Trauma Emerg Surg. 2010;36:515-9. doi: 10.1007/s00068-0100061-6.

6. Klammer G, Kadakia AR, Joos DA, Seybold JD, Espinosa N. Posterior pilon fractures: a retrospective case series and proposed classification system. Foot Ankle Int 2013;34(2):18999, doi:http://dx.doi.org/10.1177/.

7. de Vries JS, Wijgman AJ, Sierevelt IN, Schaap GR. Long-term results of ankle fractures with a posterior malleolar fragment. J Foot Ankle Surg. 2005;44:211-7.

8. Veltman ES, Halma JJ, de Gast A. Long-term outcome of 886 posterior malleolus fractures: a systematic review of the literature. Foot Ankle Surg. 2016;22:73-7. doi: 10.1016/j. fas.2015.05.003.

9. Gardner MJ, Brodsky A, Briggs SM, Nielson JH, Lorich DG. Fixation of posterior malleolar fractures provides greater syndesmotic stability. Clin Orthop Relat Res. 2006;447:165-71. doi: 10.1097/01.blo.0000203489.21206.a9.

10. Bartoníček J, Rammelt S, Tuček M, Naňka O. Posterior malleolar fractures of the ankle. Eur J Trauma Emerg Surg. 2015;41:587-600. doi: 10.1007/s00068-015-0560-6.

11. Macko VW, Matthews LS, Zwirkoski P, Goldstein SA. The joint-contact area of the ankle: the contribution of the posterior malleolus. J Bone Joint Surg Am. 1991;73:347-51.

12. Boraiah S, Kemp TJ, Erwteman A, Lucas PA, Asprinio DE. Outcome following open reduction and internal fixation of open pilon fractures. J Bone Joint Surg Am. 2010;92:346-52. doi: 10.2106/JBJS.H.01678.

13. Drijfhout van Hooff CC, Verhage SM, Hoogendoorn JM. Influence of fragment size and postoperative joint congruency on long-term outcome of posterior malleolar fractures. Foot Ankle Int. 2015;36:673-8. doi: 10.1177/1071100715570895.

14. Assal M, Ray A, Stern R. Strategies for surgical approaches in open reduction internal fixation of pilon fractures. J Orthop Trauma. 2015;29:69-79. doi: 10.1097/ BOT.0000000000000218. 